\title{
FINITE POWER-ASSOCIATIVE DIVISION RINGS
}

\section{KEVIN MCCRIMMON ${ }^{1}$}

The classical Wedderburn theorem [5, p. 37] states that any finite associative division ring is a (commutative) field. A. A. Albert generalized this to finite strictly power-associative division rings of characteristic $\neq 2$. His proof used the classification of central simple Jordan algebras and proceeded by case-checking (types A, B, C, D in $[1$, p. 301] and type $E$ in $[2$, p. 11]). The purpose of this paper is to give a uniform proof of his results.

Throughout the paper all algebras will be nonassociative algebras over a field $\Phi$ of characteristic $\neq 2$; since simple rings (in particular, division rings) are simple algebras over their centroids there is no loss in generality in restricting ourselves to algebras. An algebra is a division algebra if left and right multiplications by a nonzero element are bijections; for finite-dimensional algebras this is equivalent to the nonexistence of proper zero divisors. Following N. Jacobson, we define a Jordan division algebra to be a commutative Jordan algebra with identity element such that every nonzero element $x$ is regular with Jordan inverse $y: x y=1, x^{2} y=x$. For special algebras the inverse is just the usual inverse in the associative sense. An algebraic Jordan algebra is a Jordan division algebra if and only if each nonzero $x$ generates a subfield $\Phi[x]$, the inverse being a polynomial in $x[7$, p. 1157). (Note that this condition is weaker than being a division algebra-if $\mathfrak{Q}$ is an associative quaternion division algebra then $\mathfrak{Q}^{+}$ is a Jordan division algebra with zero divisors).

The following lemma is due to Albert [1, p. 299].

Lemma 1. A finite-dimensional strictly power-associative algebra which is a division algebra contains an identity element.

Proof. Any nonzero element is nonnilpotent, so the finite-dimensional associative subalgebra it generates contains an idempotent. If $e$ is an idempotent in our algebra $\mathfrak{D}$ we have a Peirce decomposition $[3$, p. 560$]$

$$
\begin{aligned}
\mathfrak{D} & =\mathfrak{D}_{1}+\mathfrak{D}_{1 / 2}+\mathfrak{D}_{0}, \\
\mathfrak{D}_{1 / 2} & =\{x \mid e x+x e=x\}, \\
\mathfrak{D}_{i} & =\{x \mid e x=x e=i x\} \quad(i=0,1) .
\end{aligned}
$$

Received by the editors February 28, 1966.

1 This research was supported by an AFOSR Postdoctoral Fellowship. 
If $\mathfrak{D}$ is a division algebra clearly $\mathfrak{D}_{0}=0$. If $[x, y, z]$ denotes the associator $(x y) z-x(y z)$, the associativity of third and fourth powers is given by,

$$
[x, x, x]=0, \quad\left[x^{2}, x, x\right]=0 .
$$

By strict power-associativity we may linearize the latter to obtain

$$
\left[x^{2}, x, y\right]+\left[x^{2}, y, x\right]+[x y+y x, x, x]=0 .
$$

Setting $y=e, x \in \mathfrak{D}_{1 / 2}$ we obtain

$$
\begin{aligned}
0 & =\left[x^{2}, x, e\right]+\left[x^{2}, e, x\right] \\
& =\left(x^{2} x\right) e+\left(x^{2} e\right) x-x^{2}(x e+e x) \\
& =\left(x^{2} x\right) e
\end{aligned}
$$

since $x^{2} \epsilon \mathfrak{D}_{1}+\mathfrak{D}_{0}=\mathscr{D}_{1}$ by the Peirce relations $[3, \mathrm{p} .559]$. This is impossible in a division algebra unless $x=0$, so $\mathfrak{D}_{1 / 2}=0$ and $e$ is the identity for $\mathfrak{D}=\mathfrak{D}_{1}$.

The next lemma is also Albert's [1, p. 300].

LEMмA 2. A commutative strictly power-associative algebra with identity such that each nonzero element $x$ generates a finite separable extension $\Phi[x]$ of $\Phi$ is necessarily a Jordan division algebra.

Proof. By assumption it is algebraic and each nonzero element $x$ contains a (Jordan) inverse in $\Phi[x]$, so it remains only to verify the Jordan identity

$$
\left[x^{2}, y, x\right]=0 .
$$

Since $\Phi[x]$ is separable there is an extension $\Omega$ of $\Phi$ in which $x$ splits into a linear combination $x=\sum \omega_{i} e_{i}$ of orthogonal idempotents, so (2) becomes $\sum \omega_{i}^{2} \omega_{j}\left[e_{i}, y, e_{j}\right]=0$. Now the terms $\left[e_{i}, y, e_{i}\right]$ vanish by commutativity, and since the algebra obtained by extending the base field is still strictly power-associative it suffices to prove

$$
\left[e, y, e^{\prime}\right]=0
$$

for orthogonal idempotents $e, e^{\prime}$ in any commutative strictly powerassociative algebra $\mathfrak{D}$.

Corresponding to the decomposition (1) relative to $e$ we have a decomposition

$$
\mathfrak{D}=\mathfrak{D}_{1}^{\prime}+\mathfrak{D}_{1 / 2}^{\prime}+\mathfrak{D}_{0}^{\prime}
$$

relative to $e^{\prime}$. The Peirce relations $[3$, p. 559], $[4$, p. 505] and $[8, \mathrm{pp}$. 366-367] imply 


$$
\begin{gathered}
\mathfrak{D}_{i}^{2} \subset \mathfrak{D}_{i}, \mathfrak{D}_{i} \mathfrak{D}_{j}=0, \mathfrak{D}_{i} \mathfrak{D}_{1 / 2} \subset \mathfrak{D}_{1 / 2}+\mathfrak{D}_{j} \quad(j=1-i, i=0,1) \\
z \in \mathfrak{D}_{i} \Rightarrow U_{z} \mathfrak{D}_{1 / 2} \subset \mathfrak{D}_{j} \quad\left(U_{z}=2 L_{z}^{2}-L_{z^{2}}\right)
\end{gathered}
$$

(and dually for $\mathfrak{D}_{1}^{\prime}, \mathfrak{D}_{1 / 2}^{\prime}, \mathfrak{D}_{0}^{\prime}$ ). We have

$$
\mathfrak{D}_{1 / 2}+\mathfrak{D}_{1} \subset \mathfrak{D}_{1 / 2}^{\prime}+\mathfrak{D}_{0}^{\prime}
$$

(and dually) because the $\mathfrak{D}_{1}^{\prime}$ component $x_{1}^{\prime}$ of $x=x_{1 / 2}+x_{1}\left(x_{i} \in \mathfrak{D}_{i}\right)$ is $U_{e^{\prime}} x=U_{e^{\prime}} x_{1 / 2} \in \mathfrak{D}_{1}$ by (4) since $e^{\prime} \in \mathfrak{D}_{0}$, so $x_{1}^{\prime} \in \mathfrak{D}_{1}^{\prime} \cap \mathfrak{D}_{1}$, hence $x_{1}^{\prime}$ $=e^{\prime} x_{1}^{\prime} \in \mathfrak{D}_{0} \mathscr{D}_{1}=0$.

Since $e \in \mathscr{D}_{1}, e^{\prime} \in \mathscr{D}_{0}$, the orthogonality relations (4) imply (3) if $y \in \mathfrak{D}_{1}+\mathfrak{D}_{0}$. For $y \in \mathfrak{D}_{1 / 2}$ by $(5)$ we have $y \in \mathfrak{D}_{1 / 2}^{\prime}+\mathfrak{D}_{0}^{\prime}, y e^{\prime} \in \mathfrak{D}_{1 / 2}^{\prime} \subset \mathfrak{D}_{1 / 2}$ $+\mathfrak{D}_{0}$; but $y e^{\prime} \in \mathfrak{D}_{1 / 2} \mathfrak{D}_{0} \subset \mathfrak{D}_{1 / 2}+\mathfrak{D}_{1}$ by (4), so $y e^{\prime} \in \mathfrak{D}_{1 / 2}$. From this we see $\left[e, y, e^{\prime}\right]=\left(\frac{1}{2} y\right) e^{\prime}-\frac{1}{2}\left(y e^{\prime}\right)=0$, and (3) is proved in all cases.

The following lemma is well known.

Lемма 3. A commutative alternative ring without nonzero nilpotent elements is associative.

Proof. We will show $[x, y, z]^{3}=0$ for all $x, y, z$. We have $3[x, y, z]$ $=[x, y, z]-[x, z, y]+[z, x, y]=[x y, z]+x[z, y]+[z, x] y=0$ by alternativity and commutativity, so associators are annihilated by 3 . By Artin's Theorem [9, p. 29] any subring generated by two element is a commutative associative ring, so $(u-v)^{3}=u^{3}-v^{3}$ $-3(u-v) u v$ and $(u v)^{3}=u^{3} v^{3}$. Setting $u=(x y) z, v=x(y z)$ in the first of these we have $u-v=[x, y, z]$, so $3(u-v)=0$ by the above, and $[x, y, z]^{3}=\{(x y) z\}^{3}-\{x(y z)\}^{3}$. Using the second relation this becomes $\left(x^{3} y^{3}\right) z^{3}-x^{3}\left(y^{3} z^{3}\right)=\left\{x\left(x y^{3}\right) x\right\} z^{3}-x\left\{x\left(y^{3} z^{3}\right) x\right\}=x\left\{\left(x y^{3}\right)\left(x z^{3}\right)\right\}$ $-x\left\{\left(x y^{3}\right)\left(z^{3} x\right)\right\}=0$ by the Moufang identities [9, p. 28].

Finally, we come to a lemma of J. M. Osborn. A *-simple ring is a ring with an involution $*$ which has no proper $*$-invariant ideals.

Lemma 4. $A$ *-simple associative ring with involution generated by its symmetric elements and such that the nonzero symmetric elements are invertible is either a division ring or a direct sum of two anti-isomorphic division rings.

Proof. Let 3 r be the set of elements without right inverses, $3 l$ those without left inverses, $B=Z_{r} \cup Z_{l}$ the singular elements, and $\mathfrak{W}=\mathfrak{S}(\mathfrak{A}, *)$ the symmetric elements of our ring $\mathfrak{A}$ under the involution $*$. We claim

$$
3 r=3 \imath=3 .
$$

It suffices to show $3_{r} \subset Z_{l}$. If $z \in Z_{r}$ then $z z^{*} \in Z_{r} \cap \$$, so by assump- 
tion $z z^{*}=0$. Since $z$ is a left zero divisor it can't have a left inverse, and $z \in 3 l$.

If $\mathbb{Z}=0, \mathfrak{A}$ is a division ring.

Suppose $3 \neq 0$. Now $3^{*}=3$, and from (6) we have

$$
\mathfrak{A} 3 \subset \mathfrak{3}, \quad 3 \mathfrak{Z} \subset 3 .
$$

By $*$-simplicity 8 cannot be an ideal, so there must be $z, w \in B$ with $z+w \notin 3$. If $z+w=x$ is invertible we have $e+f=1$ for $e=z x^{-1}$, $f=w x^{-1} \in \mathfrak{B}$. By (7) $e$ and $e^{*}$ are orthogonal (e.g., $e e^{*} \in \mathfrak{Z} \cap \mathfrak{S}=0$ ), so $e^{*}=e^{*} 1=e^{*} f$; similarly $f^{*}=f^{*} e$, and applying the involution gives $f=e^{*} f=e^{*}$, so $1=e+e^{*}$ is a sum of two or thogonal idempotents in 3 . By (7) $e \mathfrak{S C} e^{*}$ and $e^{*} \mathfrak{S} e$ are contained in $\mathfrak{Z} \cap \mathfrak{S}$, so $e \mathfrak{S c} e^{*}=e^{*} \mathfrak{S} e=0$. Thus $\mathfrak{S}=1 \mathfrak{S} 1 \subset e \mathfrak{A} e+e^{*} \mathfrak{U} e^{*}$. By assumption $\mathfrak{E}$ generates $\mathfrak{A}$, so $\mathfrak{A}=\mathfrak{B} \oplus \mathfrak{B}^{*}$ for $\mathfrak{B}=e \mathfrak{A} e$. Clearly the nonzero elements of $\mathfrak{B}$ must be invertible, so $\mathfrak{A}$ is a direct sum of two anti-isomorphic division rings.

Now we put the results together.

ThEOREM 1. A Jordan division ring of characteristic $\neq 2$ generated by two elements is either of the form $\Delta^{+}$for $\Delta$ an associative division ring or $\mathfrak{S}(\Delta, *)$ for $\Delta$ an associative division ring with involution.

The methods of Shirshov and Cohn (see [6, p. 207]) show that such a ring, being generated by two elements, is isomorphic to $\mathfrak{S}(\mathfrak{A}, *)$ for $\mathfrak{A}$ an associative ring with involution. We may assume $\mathfrak{A}$ is generated by its symmetric elements, and since a maximal $*$-invariant ideal $\mathfrak{M}$ induces an isomorphism of the (simple) Jordan division ring on to $\mathfrak{S}(\mathfrak{U}, *)$ where $\overline{\mathfrak{U}}=\mathfrak{A} / \mathfrak{M}$ is $*$-simple we may as well assume from the start that $\mathfrak{A}$ is $*$-simple. Thus we can apply Lemma 4 to conclude $\mathfrak{U}=\Delta$ or $\mathfrak{A}=\Delta \oplus \Delta^{*}$, and in the latter case $\mathfrak{S}(\mathfrak{A}, *)$ is isomorphic to $\Delta^{+}$.

For the next theorem we remark that the actual construction of $\Delta$ (see [6]) shows that $\Delta$ is finite-dimensional (or finite) if the Jordan ring is finite-dimensional (or finite).

ThEOREM 2. A finite Jordan division ring of characteristic $\neq 2$ is a finite (commutative, associative) field.

By Lemma 3 it suffices to prove the ring is alternative, i.e., that every subring generated by two elements is associative, and this follows from Theorem 1 since the finite division ring of Theorem 1 is a (commutative, associative) field by Wedderburn's theorem and the Jordan ring is a subfield.

THEOREM 3. A finite strictly power-associative ring which is a division ring of characteristic $\neq 2$ is a finite (commutative, associative) field. 
By Lemma 1 such an algebra $\mathfrak{D}$ contains an identity element. Each nonzero element $x$ generates a finite extension $\Phi[x]$ of the centroid $\Phi$ which is separable since $\Phi$ is finite. Since passage to the symmetrized algebra does not affect multiplication in $\Phi[x]$, Lemma 2 shows $\mathfrak{D}^{+}$ is a finite Jordan division algebra. By Theorem $2 \mathfrak{D}^{+}$is a finite Jordan division algebra. By Theorem $2 \mathfrak{D}^{+}$is a finite field, hence a finite separable extension of $\Phi$. By the theorem of the primitive element $\mathfrak{D}^{+}=\Phi[x]$. But then $\mathfrak{D}=\Phi[x]$ is again a field.

\section{REFERENCES}

1. A. A. Albert, On nonassociative division algebras, Trans. Amer. Math. Soc. 72 (1952), 296-309.

2. - - A construction of exceptional Jordan division algebras, Ann. of Math. (2) 67 (1958), 1-28.

3. - Power associative rings, Trans. Amer. Math. Soc. 64 (1948), 552-593.

4. - A theory of power associative commutative algebras, Trans. Amer. Math. Soc. 69 (1950), 503-527.

5. E. Artin, Geometric algebra, Interscience, New York, 1957.

6. N. Jacobson: Associative algebras with involution and Jordan algebras, Nederl. Akad. Wentensch. Proc. Ser. A 69 (1966), 202-212 (Indag. Math. 28).

7. - A coordinatization theorem for Jordan algebras, Proc. Nat. Acad. Sci. U.S.A. 7 (1962), 1154-1160.

8. L. Kokoris, New results on power-associative algebras, Trans. Amer. Math. Soc. 77 (1954), 363-373.

9. R. D. Schafer. An introduction to nonassociative algebras, Academic Press, New York, 1966.

Massachusetts Institute of Technology 